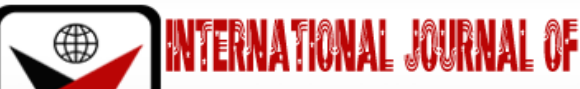

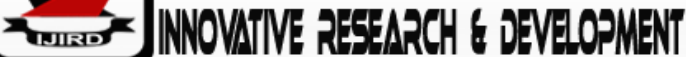

ISSN 2278-0211 (Online)

\section{Mathematical Modelling and Optimal Controls for Controlling Pneumonia-HIV Co-Infection}

\author{
Nebert Kituni Wafula \\ Graduate Assistant, Department of Mathematics, Kibabii University, Kenya \\ Boniface Otieno Kwach \\ Lecturer, Department of Mathematics, Kibabii University, Kenya \\ Vincent Nyongesa Marani \\ Lecturer, Department of Mathematics, Kibabii University, Kenya
}

\begin{abstract}
:
Bacterial infections like Pneumonia have emerged as an important cause of morbidity and mortality in individuals infected with HIV giving rise to their co-infection. There are mathematical models that describe; the co-dynamics, treatment and protection mechanisms of Pneumonia and HIV infections. Therefore, this research is aimed at determining optimal control treatments through developing a deterministic mathematical model Pneumonia-HIV coinfection incorporating the use of anti-pneumonia and ART treatment interventions as controls. The uncontrolled pneumonia-HIV co-infection is presented with its analysis. Finally, the optimal control theory for Pneumonia-HIV coinfection model is derived analytically by applying the Pontryagin's Maximum Principle.
\end{abstract}

Keywords: Antiretroviral therapy, antiretroviral, pontryagin's maximum principle, basic reproduction number

\section{Introduction}

Human-Immunodeficiency Virus (HIV) is a virus that causes acquired-immunodeficiency syndrome (AIDS). It targets the immune system, specifically the CD4 cells or T cells of the infected individual weakening it and making an individual incapable of resisting attack from a wide range of infections (WHO, 2012). Currently, there is no cure or vaccination for HIV infected individuals but HIV Testing Services are being offered to people with HIV and AIDS to help in improving their quality of lives as well as controlling the spread. The general treatment of HIV positive people is with ARV and HAART drugs which can lower the viral load set point while prolonging the life of infected person (Ho, 1995). This treatment reduces the risk of HIV transmission. But before administering the ARV, an individual is first administered with prophylaxis for opportunistic, counseling and assessing the eligibility of ARV. Regardless of ART or HAART treatment, the infectiousness is not restored to susceptibility or reduced and hence an infected individual could still cause the infection. Research shows that there are millions approximately 34 million of individuals who have HIV and AIDS and died from the disease (UNAIDS, 2013; WHO, 2014). This is evident that further studies and researches on the disease are needed in order to reduce the number of infections.

On the other hand, Pneumonia is an infectious disease that is caused by microbes including bacteria, virus, and fungi among people with severely weakened immune systems or example those infected with HIV. It is classified as an airborne disease more generally a lung infection involving alveoli (air sacs). The common causes of pneumonia are Streptococcus Pneumonae (bacterial), Pneumocystis Carinii Pneumonia (PCP) and histoplasma ( fungal) and Haemophilus influenza type $b$ and respiratory syncytial virus (RSV) (viral) (Gray and Zar, 2010; Greenwood ,2000). Currently, there is no specific effective prevention, treatment or vaccination of Pneumonia as its cause has several factors. Generally the disease can be vaccinated with pneumococcal conjugate vaccines or treated with antibiotics (cotrimoxazole prophylaxis, amoxillin, ampicillin and gentamycine dispersible tablets).

The co-infection due to Pneumonia and HIV results once the immune system has been impaired with HIV in which CD4 or T-cells are less than 200 cells per millimetre cubed giving a chance to Pneumonia infection. Research shows that the lungs are the principal target of HIV associated complications and persons with HIV infections are at an increased risk for a wide spectrum of opportunistic cases of pneumonia, neoplasms and pulmonary condition (WHO, 2012; Sogaard, et al , 2008). It has become a burden to the society disrupting the social-economical systems as efforts have been directed to curb these instances.

Several scholars have developed mathematical models which play a great role in proposing ways of controlling the transmission co-dynamics of HIV and pneumonia (Lutera et al., 2018; J. Nthiiri et al., 2015; K. Nthiiri et al., 2016; Onyinge et al., 2016). 
These models did not incorporate the aspect of optimal controls. Therefore, this study aims at incorporating the aspect of optimal control theory (Pontryagin's Maximum Principle) through developing a mathematical model, analyzing and obtaining optimal treatment controls for co-infection of Pneumonia and HIV that that will be effective.

\section{Model Formulation}

We formulated a model for the co-infection of Pneumonia and HIV that subdivide the total population at time $t$, $N(t)$ into the following compartments:

$S(t)$ : The number of individuals that are susceptible to both Pneumonia and HIV infections. This compartment is increased by recruits at the rate, $\Lambda$ and it is increased from those individuals who have recovered from Pneumonia $R_{p}$ after losing their temporary immunity at the rate $\delta$. These individuals can get Pneumonia infection and move to the $I_{p}$ at the rate $\lambda_{p}$ and also can get HIV infection and move to the $I_{h}$ class at the rate $\lambda_{h}$. $\lambda_{h}$ and $\lambda_{p}$ are given as:

$\lambda_{p}=\frac{\phi_{1}\left(I_{p}(t)+\iota_{1} I_{p h}(t)+\iota_{2} A_{p h}(t)\right)}{N(t)}, \lambda_{h}=\frac{\phi_{2}\left(I_{h}(t)+{ }_{1} I_{p h}(t)\right)}{N(t)}$

where $\varphi_{1}$ is the effective transmission for Pneumonia and $\iota_{2}>\iota_{1}$ are modification parameters which accounts for relative increased infectiousness with Pneumonia, $\varphi_{2}$ is the effective transmission rate for HIV and $\beta_{1}$ is a modification parameter for increased relative infectiousness of individuals with HIV.

$I_{p}(t)$ : The number of individuals infected with Pneumonia. They are reduced by recoveries from Pneumonia at the rate 1 while others get infected with HIV and progress to $I_{p h}$ class at the rate $\delta_{1} \lambda_{h}$. Mortality induced by Pneumonia under $I_{p}$ class occurs at the rate $d_{p} . R_{p}(t)$ : The number of individuals who have recovered from Pneumonia infection. These individuals lose their temporary immunity and they get to susceptible class at the rate $\delta$ while others get infection from HIV at the rate $\lambda_{h}$ and progress to $I_{h}$ class.

$I_{h}(t)$ : The number of individuals infected with HIV with no clinical symptoms of HIV and AIDS who progress to the class $A_{h}$ by developing clinical symptoms of HIV and AIDS at the rate $\gamma_{1}$ while others get infected with Pneumonia and progress to $I_{p h}$ class at the rate $\delta_{2} \lambda_{p} . I_{p h}(t)$ : The number of individuals co-infected with both Pneumonia and HIV with no clinical symptoms of HIV and AIDS. These individuals recover from Pneumonia at the rate 2 and move to $I_{h}$ class, others develop HIV and AIDS symptoms and progress to $A_{p h}$ class at the rate $\gamma_{2}$ while others die from Pneumonia induced mortality rate $d_{p}$. $A_{h}(t)$ : The number of individuals infected with HIV showing clinical symptoms of HIV and AIDS. These individuals seek medication from Pneumonia and they progress to $A_{h}$ class at a recovery rate 3 and are increased from $A_{h}$ class who get infection from Pneumonia at the rate $\delta_{3} \lambda_{p}$ where $\delta_{3}>\delta_{2}$. They incur induced death from both Pneumonia and AIDS at mortality rate $d_{A p}$

$A_{p h}(t)$ : The number of individuals co-infected with both Pneumonia and HIV with clinical symptoms of HIV and AIDS. They incur AIDS induced death at the rate $d_{A}$. All classes are reduced by deaths from natural causes at the rate $\mu$.

The resulting diagram from the definition of the model is qiven in Figure 1 and described mathematically by the system (2) as;

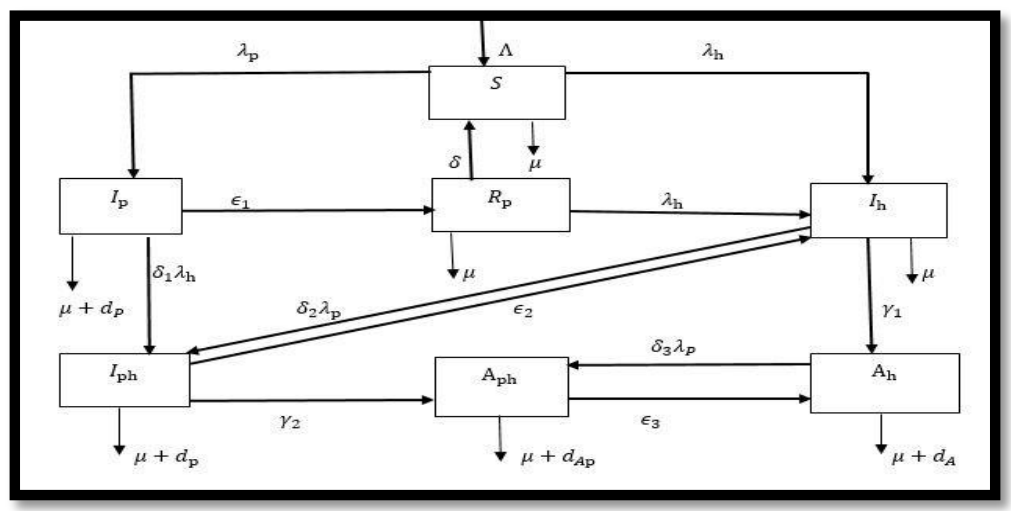

Figure 1: Flow Chart Diagram

$$
\begin{aligned}
\dot{S(t)} & =\Lambda+\delta R_{p}-\left(\lambda_{p}+\lambda_{h}+\mu\right) S \\
I_{p}(t) & =\lambda_{p} S-\left(\epsilon_{1}+\mu+d_{p}+\delta_{1} \lambda_{h}\right) I_{p} \\
R_{p}(t) & =\epsilon_{1} I_{p}-\left(\mu+\delta+\lambda_{h}\right) R_{p} \\
I_{h}(t) & =\lambda_{h} R_{p}+\epsilon_{2} I_{p h}+\lambda_{h} S-\left(\mu+\gamma_{1}+\delta_{2} \lambda_{p}\right) I_{h} \\
I_{p h}(t) & =\delta_{1} \lambda_{h} I_{p}+\delta_{2} \lambda_{p} I_{h}-\left(\gamma_{2}+\epsilon_{2}+\mu+d_{p}\right) I_{p h} \\
A_{p h}(t) & =\gamma_{2} I_{p h}+\delta_{3} \lambda_{p} A_{h}-\left(\epsilon_{3}+\mu+d_{A p}\right) A_{p h} \\
A_{h}(t) & =\gamma_{1} I_{h}+\epsilon_{3} A_{p h}-\left(\delta_{3} \lambda_{p}+\mu+d_{A}\right) A_{h}
\end{aligned}
$$

\section{Model Analysis}

Model (2) is analyzed in a feasible bounded region $\Pi$ given as: $\Pi=\left\{\left(S(t), I_{p}(t), R_{p}(t)\right.\right.$, 
$\left.\left.I_{h}(t), I_{p h}(t), A_{p h}(t), A_{h}(t)\right) \in \mathbb{R}_{+}^{7}: N(t) \leq \frac{\Lambda}{\mu}\right\}$. Since this model deals with the co-epidemic

of the two diseases in a varying human population size we assumed all state variables and parameters to be non-negative for all time $t \geq 0$ which are bounded in the region $\Pi$. As $t \rightarrow \infty$, we have

$0 \leqslant N(t) \leqslant \frac{\Lambda}{\mu}$

Therefore the model is well posed and hence biologically meaningful.

\section{Disease-Free Equilibrium Point (DFE)}

DFE $E^{0}$ is a steady-state solution for which there is no disease or infection in the population (Olaniyi and Obabiyi, 2014). To obtain the disease-free equilibrium point we set each equation of the right handside of the model system (2) equal to zero and solve hand side the system. Since there are no infections in the human populations, we set $I_{p}(t)=R_{p}(t)=$ $I_{h}(t)=I_{p h}(t)=A_{p h}(t)=A_{h}(t)=0$ and obtain the disease-free equilibrium point $E^{0}$ of the model (2) given by:

$E^{0}=\left\{S(t), I_{p}(t), R_{p}(t), I_{h}(t), I_{p h}(t), A_{p h}(t), A_{h}(t)\right\}=\left(\frac{\Lambda}{\mu}, 0,0,0,0,0,0\right)$

\section{Endemic Equilibrium Point}

Endemic equilibrium point $E^{*}$ is a steady-state solution for which there exists a constant occurrence of diseases within the population (Olaniyi and Obabiyi, 2014). To obtain the endemic equilibrium point, we set each of the right handside of the model system (2) equal to zero and solve the resultant system using mathematics. The solutions are given as:

$$
\begin{aligned}
& S^{*}(t)=\frac{-\Lambda-p}{\mu+\lambda_{h}+\lambda_{p}}, A_{h}^{*}(t)=\frac{\gamma_{2} \epsilon_{3}}{Q_{5}}\left(\frac{4}{Q_{2}}+\frac{3}{\left(\mu+d_{p} \epsilon_{1}+\delta_{1} \lambda_{h}\right) Q_{2}}\right)-\frac{\gamma_{1}\left(-\mu-A_{p}-\epsilon_{3}\right)}{Q_{5}}\left(\frac{1(\Lambda-p) h}{\delta_{2} Q_{1}}\right), I_{p}^{*}(t)= \\
& \frac{\left(\Lambda-\delta R_{p}\right) \lambda_{p}}{Q_{1}}, R_{p}^{*}(t)=\frac{-\Lambda-\delta R_{p}}{\mu+\lambda_{h}+\lambda_{p}}+\frac{\epsilon_{1}}{\lambda_{h}}\left(\frac{Q_{4}}{Q_{2}}+\frac{Q_{3}}{\left(\mu+d_{p} \epsilon_{1}+\delta_{1} \lambda_{h}\right) Q_{2}}\right)-\frac{\left(-\mu-\gamma_{1}-\delta_{2} \lambda_{p}\right)}{\lambda_{p}}\left(\frac{Q_{4}}{Q_{2}}+\left(\frac{Q_{3}}{\left(\mu+d_{p} \epsilon_{1}+\delta_{1} \lambda_{h}\right) Q_{2}}\right)\right), \\
& I_{h}^{*}(t)=-\frac{\delta_{1}\left(\Lambda-\delta R_{p}\right) \lambda_{h}}{\delta_{2} Q_{1}}+\frac{\left(-\mu-d_{p}-\gamma_{1}-\epsilon_{2}\right)}{\delta_{2} \lambda_{p}}\left(\frac{Q_{4}}{Q_{2}}+\frac{Q_{3}}{\left(\mu+d_{p} \epsilon_{1}+\delta_{1} \lambda_{h}\right) Q_{2}}\right), I_{p h}^{*}(t)=\frac{-Q_{4}}{Q_{2}}-\frac{Q_{3}}{Q_{2}\left(\mu+d_{p}+\epsilon_{1}+\delta_{1} \lambda_{h}\right)^{\prime}}, \\
& A_{p h}^{*}(t)=\frac{\gamma_{1}}{\epsilon_{3}}\left(\frac{\delta_{1}\left(\Lambda+\delta R_{p}\right) \lambda_{h}}{\delta_{2} Q_{1}}-\frac{\left(-\mu-d_{p}-\gamma_{1}-\epsilon_{2}\right)}{\delta_{2} \lambda_{p}}\left(\frac{\gamma_{2} \epsilon_{3}}{Q_{5}}\left(\frac{Q_{4}}{Q_{2}}+\frac{Q_{3}}{\left(\mu+d_{p} \epsilon_{1}+\delta_{1} \lambda_{h}\right) Q_{2}}\right)\right)+\frac{\gamma_{2}\left(-\mu-d_{A}-\delta_{3} \lambda_{p}\right)}{Q_{5}}\left(\frac{\gamma_{2} \epsilon_{3}}{Q_{5}}\right)-\right. \\
& \frac{\gamma_{1}\left(-\mu-d_{A p}-\epsilon_{3}\right)}{Q_{5}}\left(\frac{\delta_{1}\left(\Lambda-\delta R_{p}\right) \lambda_{h}}{\delta_{2} Q_{1}}\right)
\end{aligned}
$$

where, $Q_{1}=\left(\mu+d_{p}+\epsilon_{1}+\delta_{1} \lambda_{h}\right)\left(\mu+\lambda_{h}+\lambda_{p}\right), Q_{2}=\left(\mu+\lambda_{h}+\lambda_{p}\right)\left(-\delta_{2} \epsilon_{2}\left(-\delta-\mu-\lambda_{h}\right) \lambda_{p}+\right.$ $\left.\left(-\mu-d_{p}-\gamma_{1}-\epsilon_{2}\right)\left(-\delta-\mu-\lambda_{h}\right)\left(-\gamma_{1}-\mu-\delta_{2} \lambda_{p}\right)\right), Q_{3}=\left(\Lambda+\delta R_{p}\right) \lambda_{p}\left(\delta_{2} \epsilon_{1} \lambda_{h} \lambda_{p}+\delta_{1}(-\delta-\mu-\right.$ $\left.\left.\lambda_{h}\right) \lambda_{h}\left(-\mu-\gamma_{1}-\delta_{2} \lambda_{p}\right)\right), Q_{4}=\delta_{2}\left(-\Lambda-\delta R_{p}\right)\left(\delta-\mu-\lambda_{h}\right) \lambda_{h} \lambda_{p}, Q_{5}=\delta_{3} \epsilon_{3} \lambda_{p}-\left(-\mu-d_{A p}-\right.$ $\left.\epsilon_{3}\right)\left(-\mu-d_{A}-\delta_{3} \lambda_{p}\right)$

\section{The Basic Reproduction Number, $R_{0}$}

We use next generation matrix approach to determine $R_{0}$ that measures the ability of a disease reproducing itself Van den Driessche and Watmough, 2002). The two reproduction numbers at disease free equilibrium representing the two diseases are:

$$
R_{0_{P}}=\frac{\kappa_{1}}{\left(\epsilon_{1}+\mu+d_{p}\right)}, R_{0_{H}}=\frac{\left(\mu+d_{A}\right) \kappa_{2}+\gamma_{1} \text { 住 }_{2}}{\left(\mu+\gamma_{1}\right)\left(\mu+d_{A}\right)}
$$

$R_{0}=\max \left\{R_{0_{P}}, R_{0_{H}}\right\}$

Such that;

\section{Local Stability of the Disease Free Equilibrium Point}

The Jacobian matrix of the model system (2) is used to evaluate the local stability of the system at $E^{0}$. At DFE, the Jacobian matrix of equation (2) is

$$
J_{1}=\left(\begin{array}{ccccccc}
-\mu & -\frac{\kappa_{1} \Lambda}{\mu} & \delta & 0 & 0 & 0 & 0 \\
0 & -\left(\epsilon_{1}+\mu+d_{p}\right) & 0 & 0 & 0 & 0 & 0 \\
0 & \epsilon_{1} & -(\mu+\delta) & 0 & 0 & 0 & 0 \\
0 & 0 & 0 & -\left(\mu+\gamma_{1}\right) & \epsilon_{2} & 0 & 0 \\
0 & 0 & 0 & 0 & B_{1} & 0 & 0 \\
0 & 0 & 0 & 0 & \gamma_{2} & B_{2} & 0 \\
0 & 0 & 0 & \gamma_{1} & 0 & \epsilon_{3} & -\left(\mu+d_{A}\right)
\end{array}\right)
$$

where, $B_{1}=-\left(\gamma_{2}+\epsilon_{2}+\mu+d_{p}\right), B_{2}=-\left(\epsilon_{3}+\mu+d_{A p}\right)$

From (5), the trace $\operatorname{tra} J_{1}=d_{A}-4 \mu-d_{A p}-\gamma_{1}-\gamma_{2}+\delta_{1}+2 \epsilon_{1}-\epsilon_{2}-\epsilon_{3}$ which is negative and determinant is given by $\operatorname{det} J_{1}\left(E^{0}\right)=-\left(-\mu-d_{A}\right) \mu\left(-\mu-\gamma_{1}\right) \epsilon_{1}\left(-\mu-d_{p}-\delta_{1}-\epsilon_{2}-\gamma_{2}\right)\left(-\epsilon_{3}-\mu-d_{A p}\right)$ which is positive if $\left(\mu-d_{A}\right) \mu\left(-\mu-\gamma_{1}\right) \epsilon_{1}\left(-\mu-d_{p}-\delta_{1}-\epsilon_{2}-\gamma_{2}\right)\left(-\epsilon_{3}-\mu-d_{A p}\right)>0$

Therefore, the disease free equilibrium point is asymptotically stable. 
$J_{2}=\left(\begin{array}{ccccccc}b_{1} & -\frac{\kappa_{1} S^{*}}{N} & \delta & -\frac{\kappa_{2} S^{*}}{N} & 0 & 0 & 0 \\ \frac{\kappa_{1} I_{p}^{*}}{N} & b_{2} & 0 & -\frac{\kappa_{2} \delta_{1} I_{p}^{*}}{N} & -\frac{\kappa_{2} \delta \rho_{1} I_{p}^{*}}{N} & 0 & 0 \\ 0 & \epsilon_{1} & b_{3} & -\frac{\kappa_{2} R_{p}^{*}}{N} & 0 & 0 & 0 \\ \frac{\kappa_{2} I_{h}^{*}}{N} & -\frac{\delta_{2} \kappa_{1} I_{h}^{*}}{N} & \lambda_{h}^{*} & \kappa_{1} \frac{R_{p}^{*}+S^{*}}{N}+b_{4} & \epsilon_{2}-\frac{\delta_{2} \kappa_{1} \iota_{1} I_{h}^{*}}{N} & 0 & 0 \\ 0 & \delta_{1} \lambda_{h}^{*}+\frac{\delta_{2} \kappa_{1} I_{h}^{*}}{N} & 0 & \delta_{2} \lambda_{p}^{*}+\frac{\delta_{1} \kappa_{2} I_{P}^{*}}{N} & b_{5}+b_{6} & 0 & 0 \\ 0 & \frac{\delta_{3} \kappa_{1} A_{h}^{*}}{N} & 0 & 0 & \gamma_{2} & b_{7}+\frac{\delta_{3} \iota_{2} \kappa_{1} A_{h}^{*}}{N} & \frac{\delta_{3} \iota_{2} \kappa_{1} A_{p h}^{*}}{N} \\ 0 & \frac{\delta_{3} \kappa_{1} A_{h}^{*}}{N} & 0 & \gamma_{1} & 0 & \epsilon_{3}-\frac{\iota_{2} \kappa_{1} \delta_{3} A_{h}^{*}}{N} & b_{8}\end{array}\right)$

where $b_{1}=-\left(\frac{\kappa_{1} I_{p}^{*}}{N}+\frac{\kappa_{1} I_{h}^{*}}{N}+\mu\right), b_{2}=-\left(\epsilon_{1}+\mu+d_{p}+\delta_{1} \lambda_{h}^{*}\right), b_{3}=-\left(\mu+\delta+\lambda_{h}^{*}\right), b_{4}=$ $-\left(\mu+\gamma_{1}+\delta_{2} \lambda_{p}^{*}\right), b_{5}=-\left(\gamma_{2}+\epsilon_{2}+\mu+d_{p}\right), b_{6}=\frac{\delta_{2} \kappa_{1} \iota_{1} I_{h}^{*}+\delta_{2} \kappa{ }_{2} I_{1}^{*}}{N}, b_{7}=-\left(\epsilon_{3}+\mu+d_{A p}\right)$,

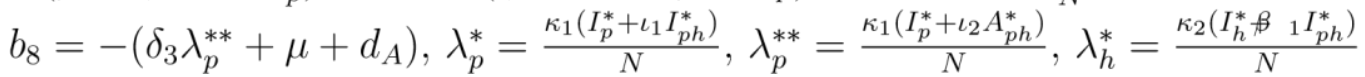

From (7), the tracetra $J_{2}=-d_{A}-\delta-7 \mu-d_{A p}-2 d_{p}-\gamma_{1}-\gamma_{2}-\epsilon_{1}-\epsilon_{2}-\epsilon_{3}-\frac{\kappa_{1} I_{h}^{*}}{N}-\frac{\kappa_{1} I_{P}^{*}}{N}+$

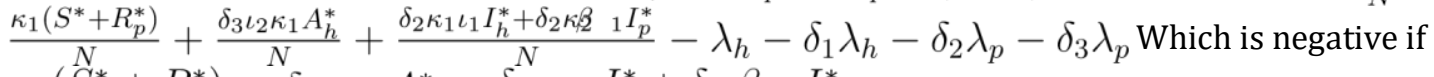
$\frac{\kappa_{1}\left(S^{*}+R_{p}^{*}\right)}{N}+\frac{\delta_{3} \iota_{2} \kappa_{1} A_{h}^{*}}{N}+\frac{\delta_{2}^{N} \kappa_{1} \iota_{1} I_{h}^{*}+\delta_{2} \kappa_{2}{ }_{1} I_{p}^{*}}{N}<0$

And determinant

$\operatorname{det} J_{2}\left(E^{*}\right)$

$\left.\left.\left.=-\frac{1}{N} \quad A_{p h} \delta_{3} \iota_{2} \kappa_{1} \epsilon_{3}-\frac{A_{h} \delta_{3} \iota_{2} \kappa_{1}}{N}\right)\right)-\frac{1}{N} \epsilon_{2}-\frac{i_{h} \delta_{1} \iota_{1} \kappa_{1}}{N}\right)\left(\frac{\delta I_{p} \epsilon_{1} \kappa_{1}}{N}+\left(-\delta-\mu-\lambda_{h}\right)\left(\frac{S I_{p} \kappa_{1}^{2}}{N^{2}}\right)\right)+$

$\left(\epsilon_{2}-\frac{I_{h} \delta_{1} \epsilon_{1} \iota_{1} \kappa_{1}}{N}\right)+\left(\frac{\delta I_{p} \epsilon_{1} \kappa_{1}}{N}+\left(-\mu-\delta-\lambda_{h}\right)\right)+\left(-\mu-\epsilon_{1}-\gamma_{2}-d_{p}\right)+\frac{S I_{p} \kappa_{2} \kappa_{1}}{N^{2}}$

$+\left(\frac{S I_{p} \kappa_{1}^{2}}{N}+\left(-\mu-\frac{I_{h} \kappa_{1}}{N}-\frac{I_{p} \kappa_{1}}{N}\right)\left(\left(-\mu-\epsilon_{1}-\delta_{1} \lambda_{h}-d_{p}\right)\right)\right)$

$\left.\left.+\left(+\frac{I_{h} \delta_{2} \iota_{1} \kappa_{1}+I_{p} \delta \beta_{1} \kappa_{2}}{N}\right)\right)\left(\frac{R_{p}^{2} \kappa_{2}^{2} \delta}{N}\left(\frac{I_{h} I_{p} \delta_{2} \kappa_{2}^{2}}{N^{2}}+\frac{\mu I_{h} \kappa_{2}^{2}}{N}+\frac{I_{h} d_{p} \kappa_{2}^{2}}{N}+\frac{I_{h} \epsilon_{1} \kappa_{2}^{2}}{N}+\frac{I_{h} \delta_{1} \kappa_{2}^{2} \lambda_{h}}{N}\right)\right)\right)$

$+\left(\frac{\left(\mu-\delta-\lambda_{h}\right)\left(\delta_{2} \kappa_{1}\right)}{N}\right)\left(\frac{\mu I_{p} \delta_{1} \kappa_{2}}{N^{2}}+\frac{S I_{p} \kappa_{2} \kappa_{1}}{N^{2}}+\frac{I_{h} I_{p} \delta_{1} \kappa_{1} \kappa_{2}^{2}}{N^{2}}+\frac{I_{P}^{2} \delta_{1} \kappa_{1} \kappa_{2}^{2}}{N^{2}}\right)+\left(\frac{\left(\mu-\delta-\lambda_{h}\right)\left(\delta_{2} \kappa_{1}\right)}{N}\right)\left(\frac{\mu I_{p} \delta_{1} \kappa_{2}}{N^{2}}\right)+$

$+\frac{I_{h} I_{p} \delta_{1} \kappa_{1} \kappa_{2}^{2}}{N^{2}}+\frac{I_{P}^{2} \delta_{1} \kappa_{1} \kappa_{2}^{2}}{N^{2}}+\left(\frac{\left(\mu-d_{p}-\epsilon_{1}-\delta_{1} \lambda_{h}\right)\left(I_{p} \kappa_{1}\right)}{N}\right)\left(\frac{S \kappa_{1}}{N}+\frac{\delta R_{p} \kappa_{1}}{N}+S \frac{I_{h} \kappa_{2}^{2}}{N^{2}}+\frac{S I_{P}{ }_{1} \kappa_{2}^{2}}{N^{2}}-\delta \delta_{2} \lambda_{p}\right)-$

$\left(\epsilon_{2}-\frac{\delta_{1} I_{h} \iota_{1} \kappa_{1}}{N}\right)\left(\frac{\delta I_{p} R_{p} \kappa_{1} \kappa_{2}}{N^{2}}-\frac{\mu \delta_{1} I_{p} \kappa_{1}}{N}-S \frac{I_{p} \kappa_{2} \kappa_{1}}{N^{2}}-\frac{S I_{h} I_{p} \delta_{1} \kappa_{2} \kappa_{1}}{N^{2}}-\frac{S I_{h}^{2} \delta_{1} \kappa_{2} \kappa_{1}}{N^{2}}\right)\left(-\delta-\mu-\lambda_{h}\right)-$

$\left.\frac{\left(-d_{A}-\mu-\delta_{1} \lambda_{p}\right)\left(\delta_{2} I_{p} \kappa_{1}\right.}{N}\right)+\left(\epsilon_{2}-\frac{\delta_{1} I_{h} \iota_{1} \kappa_{1}}{N}\right)\left(\frac{\delta I_{p} \epsilon_{1} \kappa_{1}}{N}\right)+\delta \frac{I_{p} R_{p} \kappa_{2} \kappa_{1}}{N^{2}}-\frac{S I_{h} I_{p} \delta_{1} \kappa_{2} \kappa_{1}}{N^{2}}-\frac{I_{p} I_{h} \delta_{1} \kappa_{2} \kappa_{1}}{N^{2}}$

$+\frac{I_{p}^{2} \delta_{1} \kappa_{2} \kappa_{1}}{N^{2}}+\frac{I_{p} \delta_{1} \mu \kappa_{2} \kappa_{1}}{N^{2}}+\frac{\delta I_{p} R_{p} \kappa_{1} \kappa_{2}}{N^{2}}-\left(\frac{\mu \delta_{1} I_{p} \kappa_{1}}{N}-\frac{S I_{p} \kappa_{2} \kappa_{1}}{N^{2}}-\frac{S I_{h} I_{p} \delta_{1} \kappa_{2} \kappa_{1}}{N^{2}}-\frac{S I_{h}^{2} \delta_{1} \kappa_{2} \kappa_{1}}{N^{2}}\right)(-\delta-$

$\left.\mu-\lambda_{h}\right)$

$\operatorname{det} J_{2}>0$ if

$\left(-\delta-\mu-\lambda_{h}\right)>0$

Therefore, the endemic equilibrium point is asymptotically stable provided the inequalities

(8) and (9) hold otherwise it is unstable.

\section{Extension of the Model into Optimal Control}

We incorporate the two controls interventions, $u_{1}$ : Pneumonia treatment and $u_{2}$ : HIV treatment using the concepts of optimal control theory (Pontryagin et al., 1962) to get extended model as:

$$
\begin{aligned}
\dot{S(t)} & =\Lambda+\delta R_{p}-\left(\lambda_{p}+\lambda_{h}+\mu\right) S \\
\dot{I_{p}}(t) & =\lambda_{p} S-\left(\epsilon_{1}+u_{1}+\mu+d_{p}+\delta_{1} \lambda_{h}\right) I_{p} \\
R_{p}(t) & =\left(\epsilon_{1}+u_{1}\right) I_{p}-\left(\mu+\delta+\lambda_{h}\right) R_{p} \\
I_{h}(t) & =\lambda_{h} R_{p}+\left(\epsilon_{2}+u_{1}\right) I_{p h}+\lambda S-\left(\mu+\gamma_{1}\left(1-u_{2}\right)+\delta_{2} \lambda_{p}\right) I_{h} \\
I_{p h}(t) & =\delta_{1} \lambda_{h} I_{p}+\delta_{2} \lambda_{p} I_{h}-\left(\gamma_{2}\left(1-u_{2}\right)+\left(\epsilon_{2}+u_{1}\right)+\mu+d_{p}\right) I_{p h} \\
A_{p h}(t) & =\gamma_{2}\left(1-u_{2}\right) I_{p h}+\delta_{3} \lambda_{p} A_{h}-\left(\left(\epsilon_{3}+u_{1}\right)+\mu+d_{A p}\right) A_{p h} \\
A_{h}(t) & =\gamma_{1}\left(1-u_{2}\right) I_{h}+\left(\epsilon_{3}+u_{1}\right) A_{p h}-\left(\delta_{3} \lambda_{p}+\mu+d_{A}\right) A_{h}
\end{aligned}
$$

To study the optimal control levels, the control set $U$ is considered to be Lebsque measurable and it is defined as: $U=$ $\left\{\left(u_{1}(t), u_{2}(t)\right): 0 \leq u_{1}<1,0 \leq u_{2}<1,0 \leq t \leq T\right\}$.

The proposed objective function $J$ given by: 
$J=\int_{t_{0}}^{T}\left(k_{1} I_{p}+k_{2} I_{h}+k_{3} I_{p h}+k_{4} A_{p h}+k_{5} A_{h}+\frac{1}{2} w_{1} u_{1}^{2}+\frac{1}{2} w_{2} u_{2}^{2}\right) d t$

where $k_{1}, k_{2}, k_{3}, k_{4}, k_{5}, w_{1}$ and $w_{2}$ are positive balancing coefficients (weights)which regularize the optimal control.

We need to obtain optimal controls $\left(u_{1}^{*}, u_{2}{ }^{*}\right)$ such that:

$J\left(u_{1}^{*}, u_{2}{ }^{*}\right)=\min \left\{J\left(u_{1}(t), u_{2}(t)\right): u_{1}, u_{2} \in U\right\}$

subject to the dynamical system above (10) and the control set $U$.

\section{Existence of the Optimal Control Problem}

The basic framework of an optimal control is to prove the existence of the optimal control to characterize it for optimality. The conditions that are to be met include;

- The integrand of the objective functional is convex on $U$.

- The set of controls and corresponding state variables (admissible pairs) $A$ is not empty. (iii) The control set $U$ is compact.

- There exists non-negative constants $\tau_{1}$ and $\tau_{2}$ and $\varphi>1$ such that the integrand of the objective function is bounded by $\left.\tau_{1}+\tau_{2}\left|u_{1}\right|^{2}+\left|u_{2}\right|^{2}\right)^{\phi / 2}$

- Each right-hand side of the state system is continuous and bounded above by a linear function in the state and control variables.

- We note that all the above conditions are met, there exists unique optimal controls which are unique as a result of the Lipschitz property of the state system with respect to the state variables and a priori boundedness of the state solutions (Fleming and Rishel, 2012).

\section{Characterization of Optimal Controls}

We consider the necessary conditions to determine the optimal controls given in (12) with constraint model (10) could be obtained using the Pontryagin Maximum Principle.

$H\left(S, I_{p}, R_{p}, I_{h}, I_{p h}, A_{p h}, A_{h}, u_{1}, u_{2}, \zeta_{1}, \zeta_{2}, \zeta_{3}, \zeta_{4}, \zeta_{5}, \zeta_{6}, \zeta_{7}\right)=k_{1} I_{p}+k_{2} I_{h}$

$$
+k_{3} I_{p h}+k_{4} A_{p h}+k_{5} A_{h}+\frac{1}{2} w_{1} u_{1}{ }^{2}+\frac{1}{2} w_{2} u_{2}{ }^{2}+\sum_{i=1}^{7} \zeta_{i} l_{i}
$$

where $l_{i}$ are the $i$ th state variable equations on the right-hand side of equation (10) and $\zeta_{1}, \ldots, \zeta_{7}$ are the adjoint satisfying the following theorem.

Theorem: For optimal controls $\left(u_{1}{ }^{*}, u_{2}{ }^{*}\right)$ and solution set $\left\{\left(S(t), I_{p}(t), R_{p}(t), I_{h}(t), I_{p h}(t), A_{p h}(t), A_{h}(t)\right\}\right.$ of the corresponding state system that minimizes the objective function J over $U$, there exist adjoint variables $\zeta_{1}, \ldots, \zeta_{7}$ such that:

$\dot{\zeta}_{1}(t)=\zeta_{1}\left(\lambda_{p}+\lambda_{h}+\mu\right)-\zeta_{2} \lambda_{p}-\zeta_{4} \lambda_{p}$

$\dot{\zeta}_{2}(t)=-k_{1}+\zeta_{2}\left(\epsilon_{1}+u_{1}+\mu+d_{p}+\delta_{1} \lambda_{h}\right)-\zeta_{3}\left(\epsilon_{1}+u_{1}\right)-\zeta_{5} \delta_{1} \lambda_{h}$

$\dot{\zeta}_{3}(t)=-\zeta_{1} \delta+\zeta_{3}\left(\mu+\delta+\lambda_{h}\right)-\zeta_{4} \lambda_{h}$

$\dot{\zeta}_{4}(t)=-k_{2}+\zeta_{4}\left(\mu+\gamma_{1}\left(1-u_{2}\right)+\delta_{2} \lambda_{p}\right)-\zeta_{5} \delta_{2} \lambda_{p}-\zeta_{7} \gamma_{1}\left(1-u_{2}\right)$

$\dot{\zeta}_{5}(t)=-k_{3}-\zeta_{4}\left(\epsilon_{2}+u_{1}\right)+\zeta_{5}\left(\gamma_{2}\left(1-u_{2}\right)+\epsilon_{2}+u_{1}+\mu+d_{p}\right)-\zeta_{6} \gamma_{2}\left(1-u_{2}\right)$

$\dot{\zeta}_{6}(t)=-k_{4}+\zeta_{6}\left(\epsilon_{3}+u_{1}+\mu+d_{A p}\right)-\zeta_{7}\left(\epsilon_{3}+u_{1}\right)$

$\dot{\zeta}_{7}(t)=-k_{5}+\zeta_{7}\left(\delta_{3} \lambda_{p}+\mu+d_{A}\right)-\zeta_{6} \delta_{3} \lambda_{p}$

with transversality conditions; $\zeta_{1}(T)=\zeta_{2}(T)=\zeta_{3}(T)=\zeta_{4}(T)=\zeta_{5}(T)=\zeta_{6}(T)=\zeta_{7}(T)=0$.

Furthermore, the optimal controls are represented by;

$$
\begin{array}{r}
u_{1}{ }^{*}=\max \left\{0, \min \left(1, \frac{\left(\zeta_{2}-\zeta_{3}\right) I_{p}+\left(\zeta_{4}-\zeta_{5}\right) I_{p h}+\left(\zeta_{6}-\zeta_{7}\right) A_{p h}}{w_{1}}\right)\right\} \\
u_{2}{ }^{*}=\max \left\{0, \min \left(1, \frac{\left(\zeta_{4}-\zeta_{7}\right) I_{h}+\left(\zeta_{5}-\zeta_{6}\right) I_{p h}}{w_{2}}\right)\right\}
\end{array}
$$

Proof. From the relation in

$\dot{\zeta}_{i}(t)=-\frac{\partial H}{\partial x^{i}}$

where $i=1, \ldots, 7$, we get: 
$\dot{\zeta}_{1}(t)=\zeta_{1}\left(\lambda_{p}+\lambda_{h}+\mu\right)-\zeta_{2} \lambda_{p}-\zeta_{4} \lambda_{h}$

$\dot{\zeta}_{2}(t)=-k_{1}+\zeta_{2}\left(\epsilon_{1}+u_{1}+\mu+d_{p}+\delta_{1} \lambda_{h}\right)-\zeta_{3}\left(\epsilon_{1}+u_{1}\right)-\zeta_{5} \delta_{1} \lambda_{h}$

$\dot{\zeta}_{3}(t)=-\zeta_{1} \delta+\zeta_{3}\left(\mu+\delta+\lambda_{h}\right)-\zeta_{4} \lambda_{h}$

$\dot{\zeta}_{4}(t)=-k_{2}+\zeta_{4}\left(\mu+\gamma_{1}\left(1-u_{2}\right)+\delta_{2} \lambda\right)-\zeta_{5} \delta_{2} \lambda_{p}-\zeta_{7} \gamma_{1}\left(1-u_{2}\right)$

$\dot{\zeta}_{5}(t)=-k_{3}-\zeta_{4}\left(\epsilon_{2}+u_{1}\right)+\zeta_{5}\left(\gamma_{2}\left(1-u_{2}\right)+\epsilon_{2}+u_{1}+\mu+d_{p}\right)-\zeta_{6} \gamma_{2}\left(1-u_{2}\right)$

$\dot{\zeta}_{6}(t)=-k_{4}+\zeta_{6}\left(\epsilon_{3}+u_{1}+\mu+d_{A p}\right)-\zeta_{7}\left(\epsilon_{3}+u_{1}\right)$

$\dot{\zeta}_{7}(t)=-k_{5}+\zeta_{7}\left(\delta_{3} \lambda_{p}+\mu+d_{A}\right)-\zeta_{6} \delta_{3} \lambda_{p}$

With transversality conditions; $\zeta_{1}(T)=\zeta_{2}(T)=\zeta_{3}(T)=\zeta_{4}(T)=\zeta_{5}(T)=\zeta_{6}(T)=\zeta_{7}(T)=0$

We use optimality condition $\frac{\partial H}{\partial u_{i}}=0$ to obtain the time varying optimal controls as below:

$\frac{\partial H}{\partial u_{1}}=u_{1} w_{1}+\left(\zeta_{3}-\zeta_{2}\right) I_{p}+\left(\zeta_{5}-\zeta_{4}\right) I_{p h}+\left(\zeta_{7}-\zeta_{6}\right) A_{p h}=0$

$$
\frac{\partial H}{\partial u_{2}}=u_{2} w_{2}+\left(\zeta_{4}-\zeta_{7}\right) I_{h}+\left(\zeta_{5}-\zeta_{6}\right) I_{p h}=0
$$

we obtain the following after re-arranging and simplifying;

$$
\begin{array}{r}
u_{1}{ }^{*}=\frac{\left(\zeta_{2}-\zeta_{3}\right) I_{p}+\left(\zeta_{4}-\zeta_{5}\right) I_{p h}+\left(\zeta_{6}-\zeta_{7}\right) A_{p h}}{w_{1}} \\
u_{2}{ }^{*}=\frac{\left(\zeta_{4}-\zeta_{7}\right) I_{h}+\left(\zeta_{5}-\zeta_{6}\right) I_{p h}}{w_{2}}
\end{array}
$$

Applying boundary conditions of each control we get;

$$
\begin{array}{r}
u_{1}{ }^{*}=\max \left\{0, \min \left(1, \frac{\left(\zeta_{2}-\zeta_{3}\right) I_{p}+\left(\zeta_{4}-\zeta_{5}\right) I_{p h}+\left(\zeta_{6}-\zeta_{7}\right) A_{p h}}{w_{1}}\right)\right\} \\
u_{2}{ }^{*}=\max \left\{0, \min \left(1, \frac{\left(\zeta_{4}-\zeta_{7}\right) I_{h}+\left(\zeta_{5}-\zeta_{6}\right) I_{p h}}{w_{2}}\right)\right\}
\end{array}
$$

\section{Conclusion}

The main purpose of this research work was to determine optimal controls for controlling the spread of Pneumonia and HIV co-infection. This study therefore developed a deterministic model incorporating treatment controls for the co-infection which was shown to be positively invariant as well as bounded. The Pontryagins maximum principle was used to derive and analyze the necessary conditions for the optimal controls (use of anti-Pneumonia and use of ARVs).

\section{References}

(i) Fleming, W. H.,and Rishel, R. W. (2012). Deterministic and stochastic optimal control. Springer Science and Business Media.

(ii) Gray D.M. and Zar H. J. (2010). Community-acquired Pneumonia in HIV infected children: a global perspective. Curr Opin Pulm Med. 16(3):208-16.

(iii) Greenwood B. (2000). The Epidemology of Pneumococcal Infection in Children in Developing World, The Royal Society, 354:777-785

(iv) Ho D. D. (1995). Time to hit HIV, early and hard, New England Journal of Medicine., 333(7), 450-451. //httpdx.doi.org/10.1056/nejm199508173330710.

(v) Lutera J., Mbete D. and Wangila S. (2018). Co-infection Model of HIV/AIDS Pneumonia on the Effect of Treatment at Initial and Final Stages. IOSR Journal of Mathematics (IOSR-JM), Volume 14. No. 5, 56-81.

(vi) Nthiiri J.K., Lawi G. and Manyonge A. (2015). Mathematical Modelling of Pneumonia and HIV and AIDS Co-infection the Presence of Protection. International journal Volume 9. No. 42, 2069-2085.

(vii) Nthiiri J.K., (2016). Mathematical modeling of HIV AND and AIDS coinfection with tuberculosis and pneumonia incorporating protection; PhD Thesis, Maseno University.

(viii) Olaniyi S. and O. S. Obabiyi. (2014). Qualitative analysis of malaria dynamics with nonlinear incidence function. Applied Mathematical Sciences, 8(78):3889-3904.

(ix) Onyinge O. D., Omolo N., Odundo F. (2016). Mathematical Model for Coinfection of Pneumonia and HIV/AIDS with treatment, Int. J. SCI. Eng. Appl. Sci. (IJSEAS) 2 (1)

(x) Pontryagin L. S., Boltyanskii V., Gramkrelidze R., and Mishchenko F. (1962).The Mathematical Theory of Optimal Processes, John Wiley and Sons Interscience. New York. London.

(xi) Sogaard, O., Lohse, N., Gerstoft, J., Kronborg, G., Ostergaard, L., Pedersen, C.... Obel, N. (2008). Hospitalization for Pneumonia among individuals with and without HIV infection, 1995-2007: A Danish population-based, nationwide cohort. Clinical Infectious Diseases, 47(10), 1345-1353.

(xii) UNAIDS, (2013). Global report: UNAIDS report on the global AIDS epidemic, Geneva, Swizerland. World Health Organization.

(xiii) Van den Driessche P. and Watmough J. (2002). Reproduction numbers and sub threshold endemic equilibria for compartmental models of disease transmission. Mathematical Biosciences, 180; 29-48 
(xiv) WHO (2012). HIV/AIDS.

(xv) WHO. (2014). Global HIV Report. http://www.who.int/HIV/publications/ globalreport /en. 